\title{
Article
}

\section{Discrete Optimization: The Case of Generalized BCC Lattice}

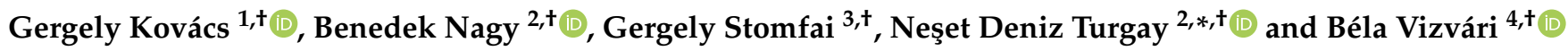 \\ 1 Department of Methodology of Applied Sciences, Edutus University, 2800 Tatabánya, Hungary; \\ kovacs.gergely@edutus.hu \\ 2 Department of Mathematics, Faculty of Arts and Sciences, Eastern Mediterranean University, \\ Famagusta 99628, North Cyprus, Turkey; benedek.nagy@emu.edu.tr \\ 3 ELTE Apáczai Csere János High School, 1053 Budapest, Hungary; 21asg@apaczai.elte.hu \\ 4 Department of Industrial Engineering, Eastern Mediterranean University, \\ Famagusta 99628, North Cyprus, Turkey; bela.vizvari@emu.edu.tr \\ * Correspondence: neset.turgay@emu.edu.tr \\ + These authors contributed equally to this work.
}

Citation: Kovacs, G.; Nagy, B.;

Stomfai, G.; Turgay, N.D.; Vizvari, B.

Discrete Optimization: The Case of Generalized BCC Lattice. Mathematics 2021, 9, 208. https://doi.org/

$10.3390 /$ math 9030208

Academic Editor: Frank Werner

Received: 26 November 2020

Accepted: 18 January 2021

Published: 20 January 2021

Publisher's Note: MDPI stays neutral with regard to jurisdictional clai$\mathrm{ms}$ in published maps and institutional affiliations.

Copyright: $\odot 2021$ by the authors. Licensee MDPI, Basel, Switzerland. This article is an open access article distributed under the terms and conditions of the Creative Commons Attribution (CC BY) license (https:// creativecommons.org/licenses/by/ $4.0 /)$.

\begin{abstract}
Recently, operations research, especially linear integer-programming, is used in various grids to find optimal paths and, based on that, digital distance. The 4 and higher-dimensional body-centered-cubic grids is the $n \mathrm{D}(n \geq 4)$ equivalent of the 3D body-centered cubic grid, a wellknown grid from solid state physics. These grids consist of integer points such that the parity of all coordinates are the same: either all coordinates are odd or even. A popular type digital distance, the chamfer distance, is used which is based on chamfer paths. There are two types of neighbors (closest same parity and closest different parity point-pairs), and the two weights for the steps between the neighbors are fixed. Finding the minimal path between two points is equivalent to an integer-programming problem. First, we solve its linear programming relaxation. The optimal path is found if this solution is integer-valued. Otherwise, the Gomory-cut is applied to obtain the integer-programming optimum. Using the special properties of the optimization problem, an optimal solution is determined for all cases of positive weights. The geometry of the paths are described by the Hilbert basis of the non-negative part of the kernel space of matrix of steps.
\end{abstract}

Keywords: integer programming; digital geometry; non-traditional grids; shortest chamfer paths; 4D grid; linear programming; optimization; digital distances; chamfer distances; weighted distances

\section{Introduction}

In digital geometry, by modeling the world on a grid, path-based distances are frequently used [1,2]. They allow to apply various algorithms of computer graphics and image processing and analysis, e.g., distance transformation [3]. Non traditional grids (related to various crystal structures) have various advantages over the traditional rectangular grids, e.g., having better packing density.

The face-centered cubic (FCC) and body-centered cubic (BCC) lattices are very important non-traditional grids appearing in nature. While the FCC grid is obtained from the cubic grid by adding a point to the center of each square face of the unit cubes, in the BCC grid, the additional points are in the centers of the bodies of the unit cells. Both FCC and BCC are point lattices (i.e., grid vectors point to grid points from any point of the grid). In this paper, we concentrate on the BCC grid and its higher dimensional generalizations. The BCC grid can be viewed as the union of the cubic lattices (the body-centers of the above-mentioned unit cubes form also a cubic lattice). The points located in edge-connected corners of a cube are called 2-neighbors, these points are from the same cubic lattice, while a corner and the body-center of a unit cell are 1-neighbors, as they are the closest neighbor point pairs in the 3D BCC lattice.

There is a topological paradox with the rectangular grids in every dimension, that can be highlighted as follows: considering a usual chessboard, the two diagonals contain 
different color squares, they go through on each other without a crossing, i.e., without a shared pixel. This is due to the fact that neighbor squares of a diagonal share only a corner point, and no side. One of the main advantages of the BCC grid is, that there are only face neighbor voxels, i.e., if two Voronoi bodies of the grid share at least one point on their boundary, then they share a full face (either a hexagon or a square). In this way, in the BCC grid, the topological paradox mentioned above cannot occur. The inner and outer part of the space is well-defined for any object built up by voxels of the BCC grid. Another important reason considering the BCC grid is its well applicability in graphical reconstruction.

The BCC lattice has been proven to be optimal for sampling spherically bandlimited signals.

To perfectly reconstruct these signals from their discrete representations, around 30\% fewer samples per unit volume have to be taken on a BCC grid than on an equivalent cubic grid. When the same number of samples is used with equivalent filters for resampling, a BCC-sampled volume representation ensures much higher quality of reconstruction than a cubic-sampled representation does [4-6].

Higher dimensional variants of FCC and BCC grids can also be defined and they have both theoretical and practical interest [7]. As their finite segments can be seen as graphs, they could also be used to build special architecture processor or computer networks. The BCC grid has the advantage that, independent of the dimension, exactly two types of neighborhoods are defined and thus, it allows relatively simple simulations (e.g., random walks) and computations. We note here that in the 4 dimensional extension of the BCC grid, the two types of neighbors have exactly the same Euclidean distance and in higher dimension, actually, the 2-neighbors of a point are closer than its 1-neighbor.

Concerning path-based (in grids, they are also called digital) distances, one of the simplest, but on the other hand, very practical and well applicable choices is to use chamfer distances [3]. These distances are, in fact, weighted distances based on various (positive) weights assigned to steps to various types of neighbors. These distances are studied in various grids [7-10] both with theoretical and practical analysis and also in connection with other fields including approximations of the Euclidean distance [11,12] and various other applications.

In this paper, similar to what we have used in other nontraditional grids (see, e.g., $[8,13,14]$ for analogous results on the semi-regular Khalimsky grid and on the regular triangular grid), we use the tools for operation research to find shortest paths (optimal solution with the terminology of operational research) between any two points. Of course, shortest (also called minimal) paths can be found in various ways, for arbitrary graphs one may use Dijkstra algorithm [15]. However, the grids we use are much more structured than arbitrary graphs as we can refer to the vertices of the graph (points of the grid) by their coordinates. Our method gives explicit formulae for the shortest paths; in this way, our method is more efficient than the application of the Dijkstra algorithm for the grids. This efficiency is obtained by the mathematical analysis of the algorithms.

Since our approach, by using operational research techniques in the field of digital geometry is not common, in the next section we describe our grids and in Section 3 we recall briefly some basic concepts of the field operations research that we need for our work. Further, in Section 4 we represent the shortest path problem on the higher dimensional $(m>3)$ BCC grids as an integer programming problem. After filtering the potential bases in Section 5, the optimal solutions are determined in Section 6. Then, in Section 7, the Gomory cut is applied to ensure integer solution. We also give some details on the Hilbert bases of rational polyhedral cones in Section 8. Finally, the paper is concluded in Section 9.

\section{The BCC Grid and Its Extensions to Higher Dimensions}

Based on a usual description of the BCC grid, we can give the following definitions used throughout of this paper. Let us consider the $m>1$ dimensional digital space $\mathbb{Z}^{m}$, especially, only the points that are represented by either only even or only odd coordinates: 
$\mathbb{B}^{m}$ is a subset of $\mathbb{Z}^{m}$ such that it includes exactly the points $\left(x_{1}, \ldots, x_{m}\right)$ if and only if $x_{1}$ $\bmod 2 \equiv x_{2} \bmod 2 \equiv \cdots \equiv x_{m} \bmod 2: \mathbb{B}^{m}=\left\{x=\left(x_{1}, \ldots, x_{m}\right) \in \mathbb{Z}^{m} \mid\right.$ the parity of all coordinates of $x$ are the same $\}$.

Note that $\mathbb{B}^{2}$ is in fact the square grid with one of its unusual representations called $2 \mathrm{D}$ diamond grid/diagonal-square grid and $\mathbb{B}^{3}$ is the original $\mathrm{BCC}$ grid, however, in this paper we use mostly $\mathbb{B}^{4}$ and its higher dimensional generalizations.

These grids can be seen as the union of two $m$ dimensional (hyper)cubic (also called rectangular) grids, they are referred as the even and odd (sub)lattices of the grid, based on the parity of the coordinates of their points.

There are two types of usually defined neighborhoods on $\mathbb{B}^{m}$ : We say that the points $\left(x_{1}, \ldots, x_{m}\right)$ and $\left(y_{1}, \ldots, y_{m}\right)$ are 1-neighbors if and only if $\left|x_{i}-y_{i}\right|=1$ for every $i \in\{1, \ldots, m\}$. These points of $\mathbb{B}^{m}$ are closest neighbors in Euclidean sense if $m \in\{2,3\}$. Moreover, in each dimension, the 1-neighbor points are the closest point pairs containing points from both the even and odd sublattices. Two points $\left(x_{1}, \ldots, x_{m}\right)$ and $\left(y_{1}, \ldots, y_{m}\right)$ of the same sublattice are 2-neighbors if and only if there is exactly one $i \in\{1, \ldots, m\}$ such that $\left|x_{i}-y_{i}\right|=2$ and the points agree on all other coordinate values, i.e., for every $j \in\{1, \ldots, m\}, i \neq j$ the equation $x_{j}=y_{j}$ holds. In case $m \in\{2,3\}$, the 2-neighbor points are the second closest point pairs of $\mathbb{B}^{m}$. However, in dimension 4 , the Euclidean distance of the two types of neighborhood relation coincide, while in higher dimensions $(m>4)$ the 2-neighbors are closer than the 1-neighbors in Euclidean sense.

Since we have two types of neighbors, we may use different weights for them in chamfer distances. The positive weights of the steps between 1- and 2-neighbors are denoted by $w$ and $u$ in this paper, respectively. We will use the term neighbor for both including 1- and 2-neighbors, and the term step as step from a point to one of its neighbors.

Then, the chamfer distance (also called weighted distance, and in this paper, we will refer to it simply with the term distance), of two points of the grid is defined as the weight of (one of the) smallest weighted path(s) between them, where a path is built up by steps to neighbor points.

Further, each of the mentioned grids is a point lattice, i.e., they are closed under addition of grid vectors. Based on that, when we look for a shortest path between two grid-points, w.l.o.g, we may assume that one of the points is the origin described by the $m$ dimensional zero vector.

\section{Theoretical Bases from Linear and Integer Programming}

\subsection{The Linear Programming Problem}

The problem of linear programming is an optimization problem such that the mathematical form of both the objective function and the constraints are linear. The standard form of the problem is as follows:

$$
\begin{gathered}
\min f^{T} x \\
G x=h \\
x \geq 0
\end{gathered}
$$

where $G$ is an $m \times n$ matrix, $h$ is an $m$ dimensional vector, $f$ is an $n$ dimensional vector of constants, and $x$ is the $n$ dimensional vector of variables. Notice that the non-negativity constraints are linear inequalities. In the practice, it is necessary to allow that some constraints are inequalities and some variables are "free", i.e., they may have positive, zero, and negative values as well. It is easy to see that any optimization problem with linear constraints and objective function can be transformed to the standard form by embedding the problem into a higher dimensional space.

\subsection{The Simplex Method and Its Geometric Content}

The linear programming and its first general algorithm, the simplex method was discovered by George Dantzig in 1947. However, it was published only in 1949 [16]. The 
simplex method is still in intensive use by professional solvers. It also gives a complete description of the convex polyhedral sets. This theory is summarized based on the book [17]. The author is well-known for the international operations research community as he obtained the EURO Gold Medal in 2003. However, this early book of him is not known for the international scientific community, although it is the best book written on the geometry of linear programming by the authors opinion.

A convex polyhedral set is the intersection of finitely many half-spaces. Notice that the ball is the intersection of infinitely many half-spaces. What is called 0-dimensional facet or corner point in the usual geometry, is called extreme point in this theory. A point is an extreme point of the polyhedral set if it is the only intersection point of the polyhedral set and a supporting hyperplane. Notice that all surface points of the ball are extreme points in this sense, as they are the intersection points of the ball and the tangent plane. The extreme points of the polyhedral set of the linear programming problem are the basic feasible solutions. For the sake of simplicity, assume that the rank of matrix $G$ is $m$. Let $B$ be a subset of the columns of $G$ such that the vectors of $B$ form a basis. The matrix formed from the elements of $B$ is an $m \times m$ matrix. It is also denoted by $B$. Assume that matrix $G$ and vector $x$ are partitioned accordingly, i.e., $G=(B, N)$, and $x^{T}=\left(x_{B}^{T}, x_{N}^{T}\right)$. The basic solution of basis $B$ is obtained if $x$ satisfies the equation system and its $x_{N}$ part is 0 . It can be obtained as follows:

$$
G x=(B, N)\left(\begin{array}{c}
x_{B} \\
x_{N}
\end{array}\right)=B x_{B}+N x_{N}=b .
$$

Hence,

$$
x_{B}=B^{-1} b-B^{-1} N x_{N} .
$$

Thus, the $x_{B}$ part of the basic solution is $B^{-1} b$. The basic solution is feasible if $B^{-1} b \geq 0$.

The simplex method starts from an extreme point of the polyhedral set, i.e., from a basic feasible solution. The algorithm moves from here to a neighboring extreme point. Two extreme points are neighboring, if a one dimensional edge of the polyhedral set connects them. The value of the objecting function in the selected neighboring extreme point is at least as good as in the current extreme point. This procedure is repeated. It stops if the current extreme point is an optimal solution. There are important consequences. One is that if an optimal solution exists, then at least one basic feasible solution is optimal. The other one is as follows: Let the set of the extreme points of the polyhedral set be the set of vertices of an undirected graph. Two vertices are connected by an edge if and only if an edge of the polyhedral set connects them. This graph is a connected graph as (i) there is no restriction on the starting point and (ii) every other extreme point can be optimal, because its support plane determines a linear objective function such that only this extreme point is optimal solution.

The optimality condition of the simplex method is as follows. Let $f_{B}$ be the vector consisting of the basic components of the objective function. The current basic feasible solution is optimal, if the inequality

$$
f_{B} B^{-1} g_{j}-f_{j} \leq 0
$$

holds for all non-basic columns of $G$. In other words, (2) must hold for all columns $g_{j}$ in $N$. If the inequality (2) is violated by a column $g_{j}$, then $g_{j}$ can replace a vector of the basis such that the objective function value either improves or remains the same. The latter case will be excluded by the strict inequalities assumed among the data of the minimal path problem, see below. The replacement can also show that the problem is unbounded. However, this case is also excluded by the assumption of the step lengths. 


\subsection{The Gomory Cut}

Gomory's method of integer programming is based on the observation that the basic variables are expressed by the other variables in the form of the equation system (1). Assume that one of these equations is

$$
x_{i}=d_{i 0}-\sum_{j \in K} d_{i j} x_{j}
$$

where $K$ is the index set of the non-basic variables. Assume further on, that $d_{i 0}$ is noninteger. It is the current value of the integer variable $x_{i}$. Let $\phi_{j}$ be the fractional part of the coefficient, i.e., the fractional part of $d_{i j}$

$$
\phi_{j}=d_{i j}-\left\lfloor d_{i j}\right\rfloor, \quad j \in K \cup\{0\} .
$$

Let us substitute these quantities into the equation. If the equation is rearranged such that all terms which are integers for sure, are in the left-hand side, the new form of the equation is obtained as follows:

$$
x_{i}-\left\lfloor d_{i 0}\right\rfloor+\sum_{j \in K}\left\lfloor d_{i j}\right\rfloor x_{j}=\phi_{0}-\sum_{j \in K} \phi_{j} x_{j} .
$$

Hence,

$$
\phi_{0} \equiv \sum_{j \in K} \phi_{j} x_{j}(\bmod 1) .
$$

All the coefficients of this relation are between 0 and 1 . Thus the two sides can be congruent only if the value of the right-hand side is in the set $\left\{\phi_{0}, \phi_{0}+1, \phi_{0}+2, \ldots\right\}$. Hence, the inequality

$$
\sum_{j \in K} \phi_{j} x_{j} \geq \phi_{0}
$$

must be satisfied. The summation in (3) goes for the non-basic variables. The value of the non-basic variables is 0 in the basic solution. Thus, this inequality is not satisfied by the current basic feasible solution as the values of the variables in the sum are all 0 . This inequality is the Gomory cut.

\section{The Integer Programming Model and Its Linear Programming Relaxation}

Many problems of combinatorial optimization are actually integer programming problems. The shortest path problem in a finite graph has also its integer programming version. A grid can be represented by an infinite graph. Therefore, the integer programming model is different.

The matrix of the steps of the 4-dimensional BCC grid are as follows:

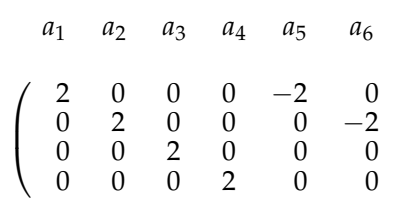

$$
\begin{array}{rrrrrrrrrrrrrrrrrr}
a_{7} & a_{8} & d_{9} & d_{10} & d_{11} & d_{12} & d_{13} & d_{14} & d_{15} & d_{16} & d_{17} & d_{18} & d_{19} & d_{20} & d_{21} & d_{22} & d_{23} & d_{24} \\
0 & 0 & 1 & 1 & 1 & 1 & 1 & 1 & 1 & 1 & -1 & -1 & -1 & -1 & -1 & -1 & -1 & -1 \\
0 & 0 & 1 & 1 & 1 & 1 & -1 & -1 & -1 & -1 & 1 & 1 & 1 & 1 & -1 & -1 & -1 & -1 \\
-2 & 0 & 1 & 1 & -1 & -1 & 1 & 1 & -1 & -1 & 1 & 1 & -1 & -1 & 1 & 1 & -1 & -1 \\
0 & -2 & 1 & -1 & 1 & -1 & 1 & -1 & 1 & -1 & 1 & -1 & 1 & -1 & 1 & -1 & 1 & -1
\end{array}
$$

The matrix of the 4D BCC grid, the vectors $a_{1}, \ldots, a_{8}$ represent steps between two neighbor points of the same sublattice (2-neighbors), while the vectors $d_{9}, \ldots, d_{24}$ represent diagonal steps, i.e., steps between neighbor points of different sublattices (1-neighbors).

This matrix is denoted by $A$. It has the same role in the particular problem of the shortest path problem as matrix $G$ in the general linear programming problem. The columns of $A$ are denoted by $a_{1}, \ldots, a_{8}, d_{9}, \ldots, d_{24}$ The first 8 columns are steps within the same rectangular grid, i.e., in the even or in the odd sublattice (2-neighbors), and the last 16 columns 
are (diagonal) steps between the two rectangular grids (1-neighbors). As we have already mentioned, the weights of these steps are denoted by $u$ and $w$. The number of columns is $2 m+2^{m}$ in the general $m$ dimensional case having vectors $a_{1}, \ldots, a_{2 m}, d_{2 m+1}, \ldots, d_{2 m+m^{2}}$, where $m \geq 2$ and is an integer. Thus, the size of the matrix is $m \times\left(2 m+2^{m}\right)$ in the general case.

It is supposed that the minimal path starts from the origin which is a point of the grid. The target point, i.e., the other end point of the path, is

$$
\mathbf{b}=\left(\begin{array}{c}
p_{1} \\
p_{2} \\
p_{3} \\
p_{4}
\end{array}\right) \text {. }
$$

Then the optimization model of the minimal path is as follows:

$$
\begin{array}{r}
\min u \sum_{j=1}^{8} x_{j}+w \sum_{j=9}^{24} x_{j} \\
\sum_{j=1}^{8} a_{j} x_{j}+\sum_{j=9}^{24} d_{j} x_{j}=\mathbf{b} \\
x_{j} \geq 0, j=1,2, \ldots, 24 \\
x_{j} \text { is integer, } j=1,2, \ldots, 24
\end{array}
$$

\section{Filtering the Potential Bases}

The aim of this paper was to give an explicit formula for the minimal path in every possible case. The theory of linear optimization and its famous algorithm called simplex method are based on the analysis of linear bases. First problems (6)-(8) are solved by the simplex method. If the optimal solution is not integer, then Gomory cut is applied for completing the solution of problems (6)-(9). However, this case occurs only once as it is shown below. The matrix $A$ has 24 columns of 4 -dimension. Thus, there are

$$
\left(\begin{array}{c}
24 \\
4
\end{array}\right)=10,626
$$

potential candidates to be a basis and producing an optimal solution. The number of candidates increases in a fast way with the dimension. It is 850,668 in 5-dimensions. Obviously, many candidates are not bases as the columns are linearly dependent. If these candidates are filtered out, then still too many candidates remain. Thus, further filtering methods must be introduced.

The grid has a highly symmetric structure. It is enough to describe the minimal paths that the two end points of the path belong to a cone such that congruent cones cover the whole 4-dimensional space without overlapping. It is assumed that the path starts from the origin which is a point of the grid and goes to the point (5) where

$$
p_{1}>p_{2}>p_{3}>p_{4}>0
$$

The assumption is

$$
p_{1}>p_{2}>\cdots>p_{m}>0
$$

in the $m$-dimensional case. 
The 4-dimensional case is discussed first. Assume that the optimal basis is $B$ which consists of 4 columns of matrix $A$. Then, a solution of the linear equation system $B \mathbf{x}=\mathbf{b}$ is required with

$$
\mathbf{x}=\left(\begin{array}{l}
x_{1} \\
x_{2} \\
x_{3} \\
x_{4}
\end{array}\right) \geq \mathbf{0}
$$

Furthermore, it must also be an integer, but this constraint is checked in a second main step. First, the bases having columns from the last 16 columns of $A$, i.e., all elements of the matrix are either +1 or -1 , are investigated. One example for such a matrix is

$$
\left(\begin{array}{rrrr}
1 & 1 & 1 & -1 \\
1 & 1 & -1 & -1 \\
1 & -1 & 1 & -1 \\
-1 & -1 & -1 & -1
\end{array}\right) .
$$

It is a basis because the columns are linearly independent and the determinant of the matrix is 8 . However, this basis does not give a non-negative solution as all coefficients in the last row are negative. Similarly, it follows from the assumptions that if two equations are added or a higher index equation is subtracted from a lower index equation, then the right-hand side of the obtained new equation is positive. Hence, to obtain a non-negative solution, it is necessary that the obtained equation has at least one positive coefficient on the left-hand side. The value of any coefficient in the new row is either +2 or 0 or -2 .

As a result of the high number of cases, we have used a computer program in Mathematica to filter out the cases not giving a non-negative solution. It investigates the four discussed conditions for every potential bases as follows. The described method can be generalized to the $m$ dimensional case.

Theorem 1. Let $S$ be a selected set of $m$ columns of matrix A. This subset is a basis and gives a non-negative solution for all right-hand sides satisfying the assumption $p_{1}>p_{2}>\cdots>p_{m}>0$ only if

1. The determinant of the basis must be non-zero.

2. Every row must contain at least one positive element, i.e., at least one +1 or +2 .

3. The sum of any two rows must contain at least one positive element, i.e., at least one +2 .

4. (Assume that the rows of matrix $A$ are indexed from 1 till $m$ such that the top row has index 1 and the index of the last row is $m$.) If a higher index row is substituted from a lower index row, then the obtained new row must contain at least one positive element, i.e., at least one +2 .

Proof. No. 1 is obvious. No. 2 follows from the fact that the original right-hand sides are positive. Nos. 3 and 4 follow again from the fact that the right-hand side remains positive after the operation of the two equations.

These four requirements produced a significant reduction of the candidates. Only 333 candidates remained from the initial

$$
\left(\begin{array}{c}
24 \\
4
\end{array}\right)=10,626
$$

$4 \times 4$ matrices. If only the last 16 columns are used, then 68 candidates remains out of 1820 ones. The latter remaining candidates are given in Appendix A Not all remaining candidates can provide with an optimal solution of the linear programming relaxation. This information is also provided in Appendix A. 


\section{The Optimal Solutions of the Linear Programming Relaxation}

Assume that $m=4$. The basis $\left\{a_{1}, a_{2}, a_{3}, a_{4}\right\}$ is always feasible if the coordinates of the target point are positive. As it was mentioned above, the simplex method can get to an optimal solution, if any, starting from any feasible solution. Therefore the analysis is started from this basis for the sake of convenience.

Lemma 1. The basis $\left\{a_{1}, a_{2}, a_{3}, a_{4}\right\}$ is optimal if and only if

$$
2 u-w \leq 0
$$

Proof. The inverse of the matrix of the basis is $\frac{1}{2} I_{4}$ where $I_{4}$ is the $4 \times 4$ unit matrix. Let $a_{j}$ be a non-basic column of matrix $A$, i.e., $j \in\{5,6, \ldots, 24\}$. The components of $a_{j}(5 \leq j \leq 8)$ or $d_{j}(9 \leq j \leq 24)$ are denoted by $a_{1 j}, a_{2 j}, a_{3 j}$, and $a_{4 j}$. The optimality condition (2) is

$$
\frac{u}{2} \sum_{i=1}^{4} a_{i j}-w \leq 0
$$

Thus, (13) can be violated only if

$$
\sum_{i=1}^{4} a_{i j}>0
$$

Condition (14) is satisfied by the vectors $d_{9}, d_{10}, d_{11}, d_{13}$, and $d_{17}$. The left-hand side of (13) is $2 u-w$ in case of vector $d_{9}$ and is $u-w$ for the four other vectors. As $u$, and $w$ are positive, the condition $u-w>0$ is stricter than $2 u-w>0$. It means that if $2 u-w>0$, then (13) is violated by the column of $d_{9}$, i.e., the basis is not optimal. If $2 u-w \leq 0$, then (13) is true for all columns.

It follows from the lemma that if $2 u-w>0>u-w$, then the only possible change of the basis is that $d_{9}$ enters instead of $a_{4}$. If $2 u-w>u-w \geq 0$, then $d_{9}$ still may enter the basis instead of $a_{4}$. If $u-w>0$, the four other vectors may enter such that $d_{10}$ enters instead of $a_{3}$ and each of $d_{11}, d_{13}$, and $d_{17}$ enters instead of $a_{4}$. This kind of analysis is very long as there are many alternative optimal solutions.

In the proofs of Theorems 2 and 3, the term weakest condition is used. The optimality condition of a basis is that (2) holds for every non-basic vector. The particular form of (2) is (13) in the case of the basis $\left\{a_{1}, a_{2}, a_{3}, a_{4}\right\}$. The particular form is different for other bases. Notice that even (13) depends on the vector, i.e., the column of matrix $A$. The condition depends on the step length, i.e., on $u$ and $w$ which are positive. The term weakest condition refers to that condition which can be violated in the easiest way. For example, the weakest condition of (13) is when all four $a_{i j}$ are equal to 1 . It is the case when the value of the left-hand side is the greatest possible. Similarly, there is a greatest possible value of the particular form of (2) in the discussed cases in the proofs of Theorems 2 and 3.

Here is a theorem which gives an optimal solution in $\mathrm{O}(1)$ steps.

Theorem 2. The basic feasible solution of one of the bases $\left\{a_{1}, a_{2}, a_{3}, a_{4}\right\},\left\{a_{1}, a_{2}, a_{3}, d_{9}\right\}$, $\left\{a_{1}, a_{2}, d_{9}, d_{10}\right\},\left\{a_{1}, d_{9}, d_{10}, d_{12}\right\}$, and $\left\{d_{9}, d_{10}, d_{12}, d_{16}\right\}$ is optimal.

Proof. Lemma 1 states that the basis is optimal if $2 u \leq w$. The simplex method is applied. As it was mentioned in the proof of the lemma, the weakest condition for entering the basis and improving the value of the objective function is $2 u<w$. If this condition is satisfied, then $d_{9}$ enters instead of $a_{4}$ and the basis becomes $\left\{a_{1}, a_{2}, a_{3}, d_{9}\right\}$. The calculation of the optimality condition can be carried out based on formula (2). The weakest condition of entering the bases is $3 u>2 w$. Thus, the basis $\left\{a_{1}, a_{2}, a_{3}, d_{9}\right\}$ is optimal if $2 u \geq w \geq \frac{3}{2} u$. If $w$ is just under $\frac{3}{2} u$, then $a_{10}$ may enter the basis. The basis becomes $\left\{a_{1}, a_{2}, d_{9}, d_{10}\right\}$ and is optimal if $\frac{3}{2} u \geq w \geq u$. There are alternative optimal solutions in the next two simplex 
iterations. One option is selected in the statement in both steps. The basis $\left\{a_{1}, d_{9}, d_{10}, d_{12}\right\}$ is optimal if $u \geq w \geq \frac{1}{2} u$. Finally, the basis $\left\{d_{9}, d_{10}, d_{12}, d_{16}\right\}$ is optimal if $\frac{1}{2} u \geq w$.

The potential optimal solutions and the objective function values are summarized in Table 1. The values of the not mentioned variables are 0 .

With the exception of the first solution, all other solutions are integer valued as the components of the target points are either odd, or even. This fact implies that the Gomory cut must be applied only at basis $\left\{a_{1}, a_{2}, a_{3}, a_{4}\right\}$.

Table 1. The optimal solutions, i.e., distances between $(0,0,0,0)$ and $\left(p_{1}, p_{2}, p_{3}, p_{4}\right)$.

\begin{tabular}{cccc}
\hline Basis & Variables & $\begin{array}{c}\text { The Value of the } \\
\text { Objective Function }\end{array}$ & $\begin{array}{c}\text { Optimality } \\
\text { Condition }\end{array}$ \\
\hline$\left\{a_{1}, a_{2}, a_{3}, a_{4}\right\}$ & $\begin{array}{c}x_{1}=\frac{p_{1}}{2}, x_{2}=\frac{p_{2}}{2}, \\
x_{3}=\frac{p_{3}}{2}, x_{4}=\frac{p_{4}}{2}\end{array}$ & $u \frac{p_{1}+p_{2}+p_{3}+p_{4}}{2}$ & $w \geq 2 u$ \\
\hline$\left\{a_{1}, a_{2}, a_{3}, d_{9}\right\}$ & $\begin{array}{c}x_{1}=\frac{p_{1}-p_{4}}{2}, x_{2}=\frac{p_{2}-p_{4}}{2}, \\
x_{3}=\frac{p_{3}-p_{4}}{2}, x_{9}=p_{4}\end{array}$ & $u \frac{p_{1}+p_{2}+p_{3}-3 p_{4}}{2}+w p_{4}$ & $2 u \geq w \geq \frac{3}{2} u$ \\
\hline$\left\{a_{1}, a_{2}, d_{9}, d_{10}\right\}$ & $\begin{array}{c}x_{1}=\frac{p_{1}-p_{3}}{2}, x_{2}=\frac{p_{2}-p_{3}}{2}, \\
x_{9}=\frac{p_{3}+p_{4}}{2}, x_{10}=\frac{p_{3}-p_{4}}{2}\end{array}$ & $u \frac{p_{1}+p_{2}-2 p_{3}}{2}+w p_{3}$ & $\frac{3}{2} u \geq w \geq u$ \\
\hline$\left\{a_{1}, d_{9}, d_{10}, d_{12}\right\}$ & $\begin{array}{c}x_{1}=\frac{p_{1}-p_{2}}{2}, x_{9}=\frac{p_{2}+p_{4}}{2}, \\
x_{10}=\frac{p_{3}-p_{4}}{2}, x_{12}=\frac{p_{2}-p_{3}}{2}\end{array}$ & $u \frac{p_{1}-p_{2}}{2}+w p_{2}$ & $u \geq w \geq \frac{1}{2} u$ \\
\hline$\left\{d_{9}, d_{10}, d_{12}, d_{16}\right\}$ & $\begin{array}{l}x_{9}=\frac{p_{1}+p_{4}}{p_{2}}, x_{10}=\frac{p_{3}-p_{4}}{2}, \\
x_{12}=\frac{p_{2}-p_{3}}{2}, x_{16}=\frac{p_{1}-p_{2}}{2}\end{array}$ & $w p_{1}$ & $\frac{1}{2} u \geq w$ \\
\hline
\end{tabular}

Theorem 2 can be generalized to $m$-dimension. Some technical details must be discussed before the formalization and the proof of the theorem.

The first issue is how to generate the columns of matrix $A$ in the $m$-dimensional case. As it was mentioned, the number of columns is $2 m+2^{m}$. The first $2 m$ columns are the columns of two diagonal matrices. All elements in the main diagonal are 2 , and -2 in the case of the first, and second matrices, respectively. A possible construction of the last $2^{m}$ columns is as follows. Let us write the integers from 0 to $2^{m}-1$ in increasing order by $m$ binary digits, each. Moreover, let us arrange the numbers vertically such that the top digit is the digit of $2^{m-1}$ and the lowest digit is the digit of 1 . In the final step, every vector is mapped component-wise into a vector where each component is 1 or -1 . A component 0 is mapped to 1 and a component 1 is mapped to -1 . Hence, $d_{2 m+1}=(1,1, \ldots, 1)$ and $d_{2 m+2^{m}}=(-1,-1, \ldots,-1)$. Hence, the next lemma follows immediately.

Lemma 2. Let $k$ be an integer such that $0 \leq k \leq m$. The components of vector $d_{2 m+2^{k}}$ are as follows: (a) the first $m-k$ components are 1 , and (b) the last $k$ components are -1 .

The matrix consisting of the columns $d_{2 m+2^{r}}, r=0,1, \ldots, m-1$ is

$$
\left(\begin{array}{ccccc}
1 & 1 & 1 & \cdots & 1 \\
1 & 1 & 1 & \cdots & -1 \\
& & & \cdots & \\
1 & 1 & 1 & \cdots & -1 \\
1 & 1 & -1 & \cdots & -1 \\
1 & -1 & -1 & \cdots & -1
\end{array}\right) .
$$

Lemma 3. The absolute value of the determinant of matrix (15) is $2^{m-1}$. 
Proof. If row $i$ is subtracted from row $i-1(i=2, \ldots, m)$, then the matrix

$$
\left(\begin{array}{ccccc}
0 & 0 & 0 & \cdots & 2 \\
0 & 0 & 0 & \cdots & 0 \\
& & & \cdots & \\
0 & 0 & 2 & \cdots & 0 \\
0 & 2 & 0 & \cdots & 0 \\
1 & -1 & -1 & \cdots & -1
\end{array}\right)
$$

is obtained. The determinants are equal. Furthermore, the absolute value of the determinant of matrix (16) is $2^{m-1}$.

Let $k$ be an integer such that $0 \leq k \leq m . B(k)$ denotes the basis

$$
\left\{a_{i} \mid i=1, \ldots, m-k\right\} \cup\left\{d_{2 m+2^{r}} \mid r=0,1, \ldots, k-1\right\}
$$

and its matrix as well. The matrix is as follows:

$$
\left(\begin{array}{cccc|c|cccc}
2 & 0 & \cdots & 0 & 1 & 1 & 1 & \cdots & 1 \\
0 & 2 & \cdots & 0 & 1 & 1 & 1 & \cdots & 1 \\
0 & 0 & \cdots & 2 & 1 & 1 & 1 & \cdots & 1 \\
\hline 0 & 0 & \cdots & 0 & 1 & 1 & 1 & \cdots & 1 \\
0 & 0 & \cdots & 0 & 1 & 1 & 1 & \cdots & -1 \\
0 & 0 & \cdots & 0 & 1 & 1 & 1 & \cdots & -1 \\
0 & 0 & \cdots & 0 & 1 & 1 & -1 & \cdots & -1 \\
0 & 0 & \cdots & 0 & 1 & -1 & -1 & \cdots & -1
\end{array}\right)
$$

Lemma 4. Let $k$ be an integer such that $0 \leq k \leq m$. The set of columns of matrix $B(k)$ form a basis of the m-dimensional Euclidean space.

Proof. The determinant of the matrix consisting of the columns (17) is the product of two subdeterminants. One is a diagonal matrix where all diagonal elements are 2 . The other one is a $k \times k$ matrix type (15). The determinants of both submatrices are different from zero.

Lemma 5. Let $k$ be an integer such that $0 \leq k \leq m$. All components of the basic solution of basis

$$
\left\{a_{i} \mid i=1, \ldots, m-k\right\} \cup\left\{d_{2 m+2^{r}} \mid r=0,1, \ldots, k-1\right\}
$$

is positive under the assumption of (11).

Proof. If $k=0$, then

$$
x_{i}=\frac{p_{i}}{2}>0, i=1, \ldots, m .
$$

The basic solution is determined by the equation

$$
B(k) x=b .
$$

Its solution is as follows:

$$
\begin{gathered}
x_{i}=\frac{p_{i}-p_{m-k+1}}{2} \quad i=1, \ldots m-k, \\
x_{2 m+1}=\frac{p_{m-k+1}+p_{m}}{2}
\end{gathered}
$$




$$
x_{2 m+2^{r}}=\frac{p_{m-k+r}-p_{m-k+r+1}}{2}, \quad r=1, \ldots, k-1
$$

Substituting the solution, it satisfies the equation system.

Lemma 6. The inverse of the matrix $B(1)$ is

$$
\left(\begin{array}{cccc|c}
0.5 & 0 & \cdots & 0 & -0.5 \\
0 & 0.5 & \cdots & 0 & -0.5 \\
0 & 0 & \cdots & 0.5 & -0.5 \\
\hline 0 & 0 & \cdots & 0 & 1
\end{array}\right)
$$

Let $k$ be an integer such that $2 \leq k \leq m$. The inverse of $B(k)$ is

$$
\left(\begin{array}{cccc|c|cccc}
0.5 & 0 & \cdots & 0 & -0.5 & 0 & 0 & \cdots & 0 \\
0 & 0.5 & \cdots & 0 & -0.5 & 0 & 0 & \cdots & 0 \\
0 & 0 & \cdots & 0.5 & -0.5 & 0 & 0 & \cdots & 0 \\
\hline 0 & 0 & \cdots & 0 & 0.5 & 0 & 0 & \cdots & 0.5 \\
0 & 0 & \cdots & 0 & 0 & 0 & 0 & \cdots & -0.5 \\
0 & 0 & \cdots & 0 & 0 & 0 & 0.5 & \cdots & 0 \\
0 & 0 & \cdots & 0 & 0 & 0.5 & -0.5 & \cdots & 0 \\
0 & 0 & \cdots & 0 & 0.5 & -0.5 & 0 & \cdots & 0
\end{array}\right) .
$$

Proof. The product of the two matrices is the unit matrix.

Now it is possible to formalize the generalization of Theorem 2 . The theorem gives a list of $m+1$ bases such that the basic solution of at least one basis is always optimal.

Theorem 3. The basic solution of at least one of the bases (17) is always optimal.

Proof. It follows from Lemmas 4 and 5 that each vector set of type (17) is a basis of the $m$-dimensional Euclidean space and its basic solution is feasible. The tool for investigating the optimality of these bases is formula (2).

Case 1, the basis $\left\{a_{i} \mid i=1, \ldots, m\right\}$.

The inverse of the matrix of the basis is $\frac{1}{2} I_{m}$ where $I_{m}$ is the $m \times m$ unit matrix. The basic part of the vector of the objective function, i.e., $f_{B}$, is the $m$-dimensional vector $(u, u, \ldots, u)$. Hence,

$$
f_{b} B^{-1}=(u, u, \ldots, u) \frac{1}{2} I_{m}=\frac{u}{2}(1,1, \ldots, 1) .
$$

A column $d_{2 m+r}\left(1 \leq r \leq 2^{m}\right)$ may enter to the basis and can improve the solution if

$$
\frac{u}{2}(1,1, \ldots, 1) d_{2 m+r}>w
$$

The weakest condition for entering the basis is obtained if the left-hand side is maximal. It is reached, if all components of $d_{2 m+r}$ is positive, i.e., at $d_{2 m+1}$. Condition (26) is

$$
\frac{u m}{2}>w
$$

Notice, that vectors $a_{j}(m+1 \leq j \leq 2 m)$ may not enter to the basis, because the basis becomes infeasible. 
Case 2, the basis $\left\{a_{i} \mid i=1, \ldots, m-k\right\} \cup\left\{d_{2 m+2^{r}} \mid r=0,1, \ldots, k-1\right\}$ and $1 \leq k \leq m$. The same logic is applied as in Case 1. The first $m-k$ components of $f_{b}$ are $u$ and the last $k$ components are $w$. It follows from (24) that

$$
\begin{aligned}
& f_{b} B^{-1}=(u, u, \ldots, u, w, w, \ldots, w) B(k)^{-1} \\
= & \left(\frac{u}{2}, \frac{u}{2}, \ldots, \frac{u}{2},-\frac{(m-k) u}{2}+w, 0, \ldots, 0\right) .
\end{aligned}
$$

The value of the general product $f_{b} B^{-1} g_{j}$ is $f_{b} B^{-1} a_{j}$ or $f_{b} B^{-1} d_{j}$, respectively, if $1 \leq j \leq 2 m$ or $2 m+1 \leq j \leq 2 m+2^{m}$, respectively. Recall that the necessary and sufficient condition that a basic solution is optimal, is that condition (2) holds for all index $j$. Moreover, if $j$ is the index of a basic variable, then the left-hand side of (2) is 0 . The value of $f_{j}$ is $u$ if $1 \leq j \leq 2 m$ and otherwise is $w$.

If $1 \leq j \leq m-k$, then $f_{b} B^{-1} a_{j}-f_{j}=0$, because the vector is in the basis. If $j=m-k+1$, then $f_{b} B^{-1} a_{j}-f_{j}=-(m-k) u+2 w-u$. It is non-positive if and only if

$$
w \leq \frac{(m-k+1) u}{2} .
$$

If $m-k+2 \leq j \leq m$ or $2 m-k+2 \leq j \leq 2 m$, then $f_{b} B^{-1} a_{j}-f_{j}=-u<0$. If $m+1 \leq j \leq 2 m-k$, then $f_{b} B^{-1} a_{j}-f_{j}=-2 u<0$. Finally, if $j=2 m-k+1$, then $f_{b} B^{-1} a_{j}-f_{j}=u(m-k)-2 w-u$. It is non-positive if and only if

$$
w \geq \frac{(m-k-1) u}{2} .
$$

The components of the vector $d_{j}$ are denoted by $d_{j i}$. Assume that $2 m+1 \leq j \leq 2 m+2^{m}$. In determining the maximal value of the left-hand side of (2), formula (28) is used. It follows immediately that the maximal value is achieved only if the first $m-k$ components of the column are 1 . The last $k-1$ components are indifferent as they are multiplied by 0 . Thus, the component $d_{j, m-k+1}$ is critical. The left-hand side of (2) as a function of this component is

$$
\begin{gathered}
\frac{(m-k) u}{2}-d_{j, m-k+1}\left(\frac{(m-k) u}{2}-w\right)-w \\
\quad= \begin{cases}0 & \text { if } d_{j, m-k+1}=1 \\
u(m-k)-2 w & \text { if } d_{j, m-k+1}=-1\end{cases}
\end{gathered}
$$

Thus, the left-hand side of (2) is nonpositive if $d_{j, m-k+1}=1$ or $d_{j, m-k+1}=-1$ and

$$
w \geq \frac{(m-k) u}{2} .
$$

(32) is a stronger condition than (30). Hence, the basis is optimal if

$$
\frac{(m-k+1) u}{2} \geq w \geq \frac{(m-k) u}{2} .
$$

Notice that the intervals of the conditions (27) and (33) $k=1, \ldots, m$ cover the nonnegative half-line which implies the statement.

Theorem 4. If (33) holds for $1 \leq k \leq m$, then the length of the minimal path is

$$
\frac{u\left(\sum_{i=1}^{m-k} p_{i}-(m-k) p_{m-k+1}\right)}{2}+w p_{m-k+1} .
$$

Proof. Notice that the optimal solution of the linear programming relaxation is integer valued. Formula (34) is obtained by substituting the optimal solution into the objective function. 


\section{The Application of the Gomory Cut}

The optimal solutions of the linear programming relaxation are given in (18)-(22). As all components of the target point are either odd or even, the optimal solutions given in (20)-(22) are integer. Non-integer optimal solution of the linear programming relaxation is obtained only, if the basis $\left\{a_{i} \mid i=1, \ldots, m\right\}$ is optimal and the target point is in different cubic lattice than the starting point. The linear programming optimal solution uses steps which do not change the cubic lattice. However, an integer feasible solution must contain an odd number from the last $2^{m}$ steps of matrix $A$. Hence, the inequality

$$
\sum_{i=m+1}^{m+2^{m}} x_{i} \geq 1
$$

must be satisfied.

Lemma 7. The Gomory cut is the inequality (35).

Proof. The inverse of the basis is $\frac{1}{2} I_{m}$. Assume that the Gomory cut is generated from the first equation of (7). Thus, all integer parts are $\frac{1}{2}$. Hence, the form of (3) is

$$
\sum_{i=m+1}^{m+2^{m}} \frac{1}{2} x_{i} \geq \frac{1}{2}
$$

It is equivalent to (35).

Theorem 5. If $\frac{u m}{2} \leq w$ and $p_{1}, \ldots, p_{m}$ are odd numbers, then an optimal solution is

$$
x_{i}=\frac{p_{i}-1}{2}, i=1, \ldots, m, \quad x_{2 m+1}=1, \quad x_{i}=0, \quad i=m+1, \ldots, 2 m+2^{m}, i \neq 2 m+1
$$

and the optimal value is

$$
u \sum_{i=1}^{m} \frac{p_{i}}{2}-\frac{m u}{2}+w
$$

Remark 1. The first condition is the opposite of (27) and ensures that the basis $\left\{a_{1}, \ldots, a_{m}\right\}$ is optimal.

Proof. The inequality (35) is added to the problem as an equation with a non-negative slack variables $s$ as follows:

$$
\sum_{i=m+1}^{m+2^{m}} x_{i}-s=1
$$

Thus, every column of matrix $A$ is extended by one component. This component is 0 in the case of the first $2 m$ columns and is 1 in the case of the last $2^{m}$. Furthermore, a new column is added which belongs to variable $s$. This column is the negative $m+1$-st unit vector, as variable $s$ appears only in the last equation. As it is shown below, the optimal basis of the extended problem is $\{1, \ldots, m\} \cup\{2 m+1\}$.

Notice that the products $B^{-1} g_{j}$ in formula (2) are the coordinates of vector $g_{j}$ in the current basis. Thus, (2) can be checked if the coordinates of $B^{-1} g_{j}$ are known. The inverse of the extended matrix $B$ is similar to (23).

Case 1. $m+1 \leq j \leq 2 m$. The coordinates are $\left(B^{-1} g_{j}\right)_{j}=-1$ and $\left(B^{-1} g_{j}\right)_{i}=0, i \neq j$. Hence, the left-hand side of (2) is $-2 u<0$. 
Case $2.2 m+2 \leq j \leq 2 m+2^{m}$. The coordinates are

$$
\left(B^{-1} g_{j}\right)_{i}=\left\{\begin{array}{cl}
0 & \text { if } d_{j i}=1,1 \leq i \leq m \\
-1 & \text { if } d_{j i}=-1,1 \leq i \leq m \\
1 & \text { if } i=m+1
\end{array}\right.
$$

where $d_{j i}$ is the $i$-th component of vector $d_{j}$. Hence, the left-hand side of (2) is

$$
u \sum_{i: d_{j i}=-1} 1+w-w \leq-u<0 .
$$

Case 3. $j=2 m+2^{m}+1$. It is the case of the unit vector of $s$. The coefficient of $s$ in the objective function is 0 . The coordinates are

$$
\left(B^{-1} g_{j}\right)_{i}=\left\{\begin{array}{cl}
\frac{1}{2} & \text { if } 1 \leq i \leq m \\
-1 & \text { if } i=m+1
\end{array}\right.
$$

Hence, the left-hand side of (2) is

$$
\frac{u m}{2}-w
$$

This value is nonpositive according to the conditions. Thus, the optimality condition is satisfied in all cases.

Corollary 1. If (33) holds for $1 \leq k \leq m$, then the length of the shortest path from the origin to the point $\left(p_{1}, p_{2}, \ldots p_{m}\right)^{T}$ is (34). If $\frac{u m}{2} \leq w$ and $p_{1}, \ldots, p_{m}$ are even numbers, then the length of the shortest path is

$$
u \sum_{i=1}^{m} \frac{p_{i}}{2}
$$

If $\frac{u m}{2} \leq w$ and $p_{1}, \ldots, p_{m}$ are odd numbers, then the length of the shortest path is (38).

\section{The Hilbert Basis of the Nonnegative Part of the Kernel Space}

Jeroslow reformulated an old paper of Hilbert [18] on the language of the contemporary mathematics and proved an important theorem [19]. Later Schrijver discovered the same theorem independently [20]. The Theorem 6 below contains the reformulated version of Hilbert's theorem (first statement) and the new theorem of Jeroslow and Schrijver.

Theorem 6. Let $\mathcal{C}$ be a cone determined by finitely many linear inequalities such that the coefficients of the inequalities are rational numbers in the $m$ dimensional space. Let

$$
\mathcal{S}=\mathcal{C} \cap Z^{m}
$$

be the set of the integer points in $\mathcal{C}$.

- $\quad$ There is a finite subset $\mathcal{T}$ of $\mathcal{S}$ such that for every vector $s \in \mathcal{S}$ there are non-negative integer weights $\alpha_{t}$ such that

$$
s=\sum_{t \in \mathcal{T}} \alpha_{t} t
$$

- If $\mathcal{C}$ is a pointed cone, i.e., the origin is the extreme point of $\mathcal{C}$, then there is only one set $\mathcal{T}$ with this property. 
Let $n=2 m+2^{m}$. The kernel space of the general matrix $A$ is

$$
\left\{x \in R^{n} \mid A x=0\right\} .
$$

Its non-negative part is the set

$$
\mathcal{P}=\left\{x \in R^{n} \mid x \geq 0, A x=0\right\} .
$$

$\mathcal{P}$ is a pointed cone. Let $\mathcal{H}$ be its Hilbert basis.

Although $\mathcal{H}$ seems an algebraic object, it has strong geometric and optimization meanings. Matrix $A$ has the property in any dimension that if a vector $a$ (or $d$ ) is its column, then the vector $-a$ (or $-d)$ is a column of $A$ as well. For the first look, the elements of $\mathcal{H}$ describe elementary circuits of the grid. For example, $a_{1}+d_{17}+d_{24}=0$ (see also Figure 1 ). Thus, starting from a point and making the steps of types 1,17 , and 24, the walk returns to the origin. However, there is another interpretation of the equation as follows:

$$
a_{1}+d_{17}+d_{24}=a_{1}-d_{16}-d_{9}=0
$$

implying that

$$
a_{1}=d_{16}+d_{9} .
$$

The meaning of the Equation (45) is that one step in the same rectangular sublattice is equivalent with two steps between the two sublattices (see also Figure 2). Thus, stepping in the same sublattice is better than the two steps between the sublattices if $u<2 w$.

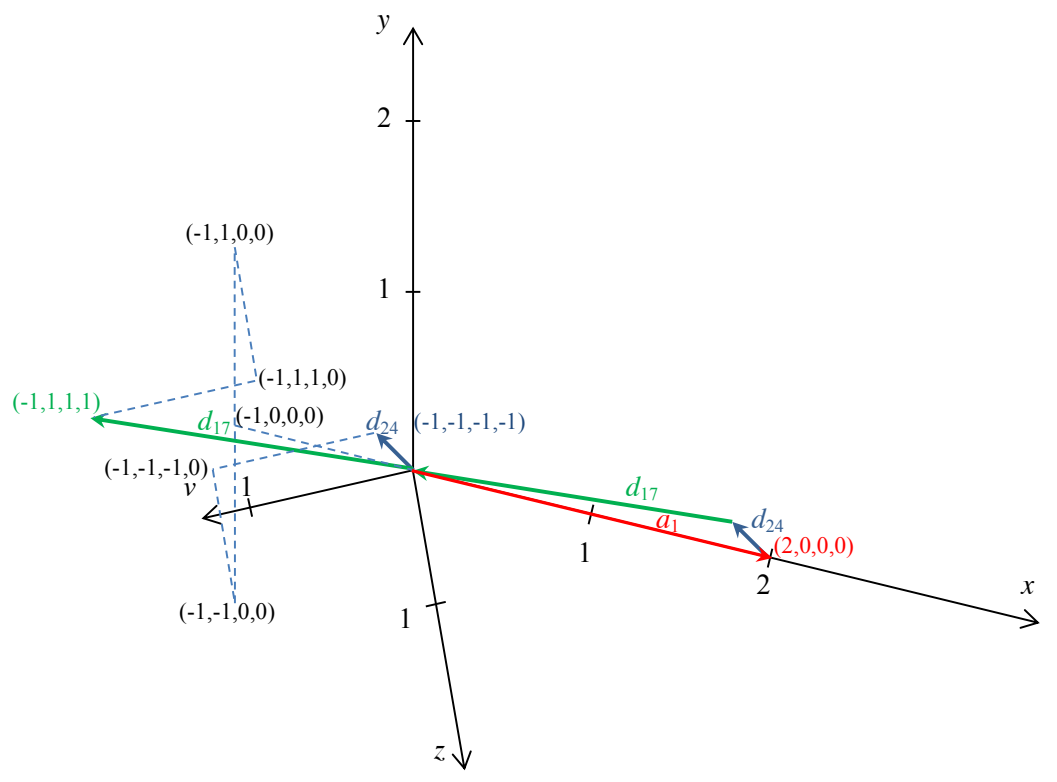

Figure 1. The sum of vectors $a_{1}, d_{17}$, and $d_{24}$ is the four-dimensional zero vector. The coordinates of the construction are also shown.

If the opposite inequality is true, i.e., $u>2 w$, then it is better to step always between the sublattices. This example has further three similar cases as follows:

$$
a_{1}=d_{10}+d_{15}, a_{1}=d_{11}+d_{14}, a_{1}=d_{12}+d_{13} .
$$

As we can see, a cycle in an undirected graph may have two different important interpretations as to walk a cycle arriving back to the initial point or to walk from a point to another in two different paths [21]. Many further composite steps can be obtained from Table 2. For example, it is possible to go from the origin to the point $(2,2,0,0)$ by using steps $a_{1}$ and $a_{2}$ with length $2 u$ or with $2 w$ by using either steps $d_{9}$ and $d_{12}$, or $d_{10}$ and $d_{11}$ as it 
is shown in Figure 3. A cycle form of the same relation based on the above-mentioned diagonal steps can be seen in Figure 4.

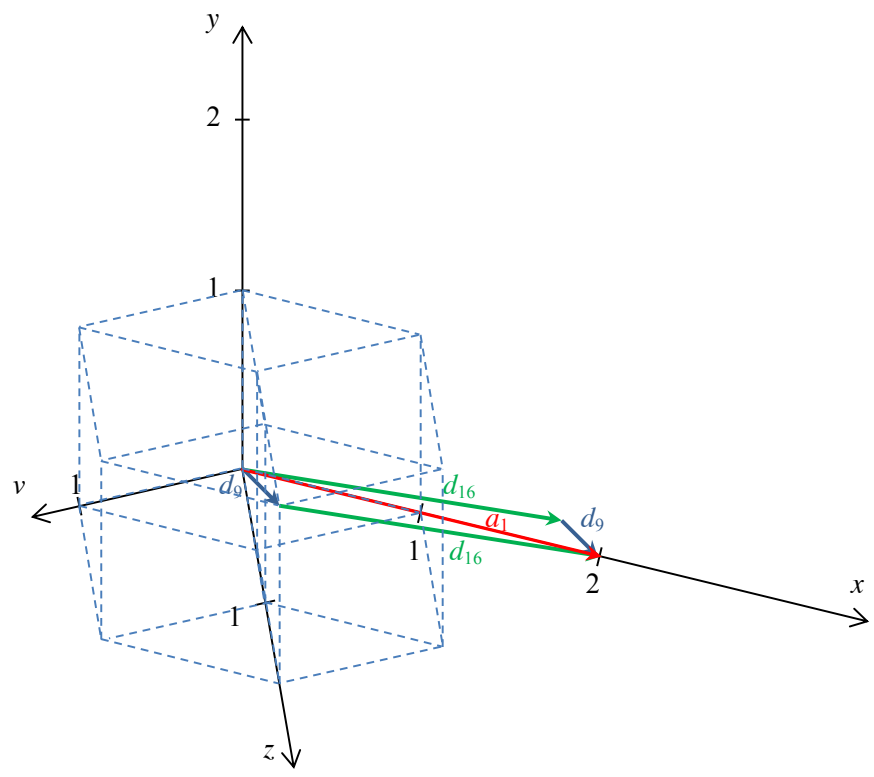

Figure 2. The sum of vectors $d_{9}$ and $d_{16}$ is exactly the vector $a_{1}$ in the four-dimensional BCC grid. A unit hypercube is also shown.

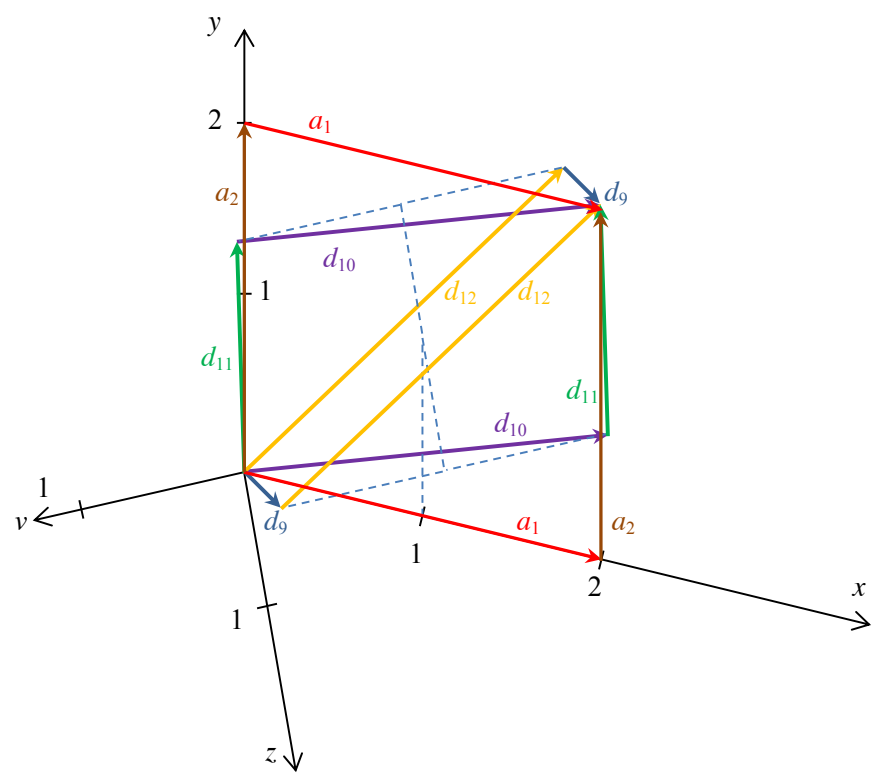

Figure 3. Alternative paths from $(0,0,0,0)$ to $(2,2,0,0)$, each is built up by two steps, in the fourdimensional BCC grid.

There is an iterative way to explore many elements of the Hilbert basis. The basic idea is that the known elements are excluded and the optimization problem looks for the next unknown element having the smallest $l_{1}$ norm. The model has variables $x_{j}$ $j \in\{1,2, \ldots, 24\}$, where the meaning of $x_{j}$ is that step $j$ is used how many times in the circuit as in (42) or (43). Thus, it must be an integer. There is a second set of variables denoted by $y_{j} j \in\{1,2, \ldots, 24\}$. They are binary variables as follows:

$$
y_{j}= \begin{cases}1 & \text { if } x_{j}>0 \\ 0 & \text { if } x_{j}=0\end{cases}
$$


The meaning of $y_{j}$ is if the step represented by the $j$ th column of matrix $A$ is included in the circuit or not.

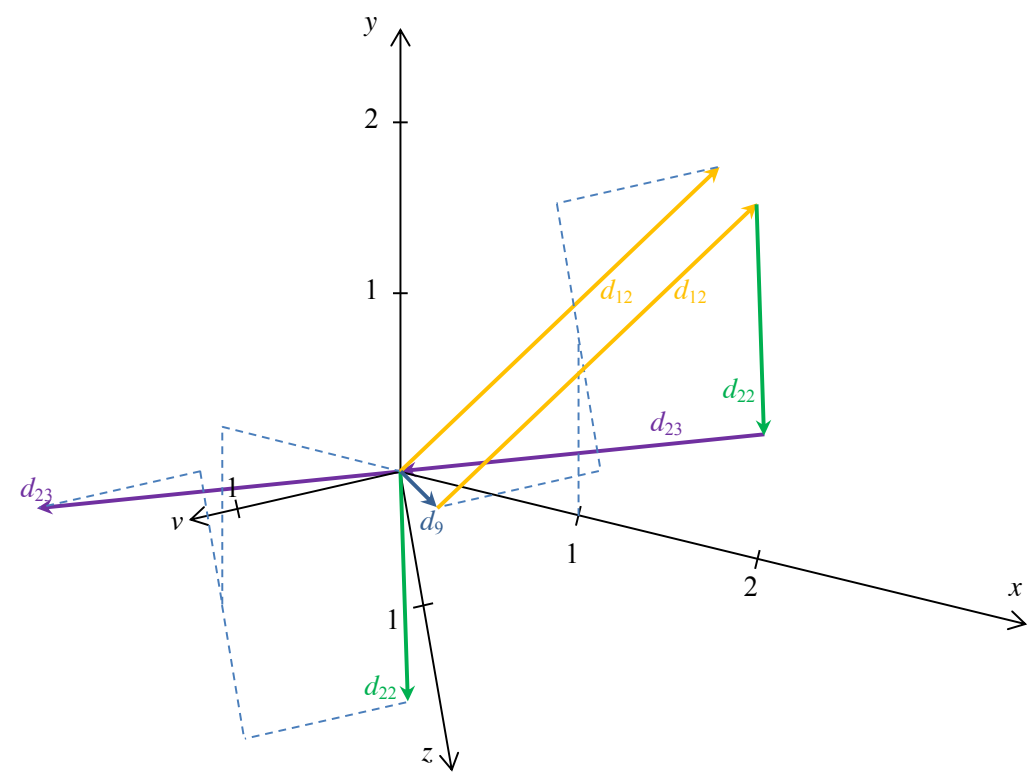

Figure 4. The sum of four diagonal vectors is the zero vector, each vector is also shown from the origin.

The objective function is the minimization of the number of used types of steps

$$
\min \sum_{j=1}^{24} y_{j}
$$

Then the constraint being in the kernel space is similar to constraint (7) but the righthand side must be the zero vector

$$
\sum_{j=1}^{8} a_{j} x_{j}+\sum_{j=9}^{24} d_{j} x_{j}=0 .
$$

The relation of the two types of variables is, that if $y_{j}=1$ if and only if $x_{j}>0$. Let $M$ be a big positive number. The constraint to be claimed is

$$
x_{j} \leq M y_{j}, j=1, \ldots, 24
$$

Notice that (50) claims only that if $x_{j}$ is positive, then $y_{j}=1$. However, it is not possible in an optimal solution that $x_{j}=0$ and $y_{j}=1$. The zero vector must be excluded by claiming that the sum of the variables must be at least 1

$$
\sum_{j=1}^{24} x_{j} \geq 1
$$

The variables must be non-negative integer and binary, respectively,

$$
x_{j} \geq 0 \text { and integer; } y_{j}=0 \text { or } 1, j=1, \ldots, 24 \text {. }
$$

The problems (48)-(52) are used in an iterative way. When a new member of the Hilbert basis is found, then it is excluded by the constraint that not all same types of steps 
can occur in a new element. Let $\bar{x}$ be the last optimal solution. Lest $S=\left\{j \mid \bar{x}_{j}>0\right\}$. Then the inequality

$$
\sum_{j \in S} y_{j} \leq|S|-1
$$

is added to the problem as a new constraint. The next optimal solution must be different from $\bar{x}$.

Some trivially existing solution, i.e., if the sum of two columns is the zero vector, can be excluded immediately. Thus, the constraints

$$
y_{1}+y_{5} \leq 1, \ldots, y_{4}+y_{8} \leq 1, y_{9}+y_{24} \leq 1, \ldots, y_{16}+y_{17} \leq 1
$$

can be introduced.

The types obtained by iterative application of the model are summarized in Table 2.

Table 2. Types of the elements of the Hilbert basis.

\begin{tabular}{ccccc}
\hline Type & \# of $\boldsymbol{a}^{\prime} \mathbf{s}$ & \# of $\boldsymbol{d}^{\prime} \mathbf{s}$ & Example & \# of Cases \\
\hline 1 & 2 & 0 & $a_{1}+a_{5}=0$ & 4 \\
2 & 0 & 2 & $d_{9}+d_{24}=0$ & 8 \\
3 & 1 & 2 & $a_{1}+d_{17}+d_{24}=0$ & 32 \\
4 & 2 & 2 & $a_{1}+a_{2}+d_{21}+d_{24}=0$ & 48 \\
5 & 0 & 4 & $d_{9}+d_{12}+d_{22}+d_{23}=0$ & 24 \\
6 & 3 & 2 & $a_{1}+a_{2}+a_{3}+d_{23}+d_{24}=0$ & 32 \\
7 & 1 & 4 & $a_{1}+d_{9}+d_{20}+d_{22}+d_{23}=0$ & 64 \\
8 & 0 & 6 & $2 \times d_{9}+d_{16}+d_{20}+d_{22}+d_{23}=0$ & 16 \\
9 & 2 & 4 & $2 \times a_{1}+d_{17}+d_{20}+d_{22}+d_{23}=0$ & 16 \\
10 & 4 & 2 & $a_{5}+a_{6}+a_{7}+a_{8}+2 \times d_{9}=0$ & 16 \\
\hline
\end{tabular}

A sequence of optimal bases of the linear relaxation is discussed in Theorem 3. The relation of the changes of the bases and the elements of the Hilbert basis is shown in Table 3. The relation can be obtained in the way shown in formula (44). Notice that the ratios of the numbers of $a$ and $d$ vectors are the same of the ratios of $u$ and $v$ where the optimal solution changes according to Theorem 2. The elements of the Hilbert basis can be obtained by taking the difference of the two optimal solutions.

Table 3. The relation of the elements of the Hilbert basis and the changes of basis.

\begin{tabular}{ccccc}
\hline Old Basis & New Basis & Hilbert Basis Element & Type & Ratio \\
\hline$a_{1}, a_{2}, a_{3}, a_{4}$ & $a_{1}, a_{2}, a_{3}, d_{9}$ & $a_{1}, a_{2}, a_{3}, a_{4}, 2 \times d_{24}$ & 10 & $\frac{4}{2}=2$ \\
\hline$a_{1}, a_{2}, a_{3}, d_{9}$ & $a_{1}, a_{2}, d_{9}, d_{10}$ & $a_{1}, a_{2}, a_{3}, d_{23}, d_{24}$ & 6 & $\frac{3}{2}$ \\
\hline$a_{1}, a_{2}, d_{9}, d_{10}$ & $a_{1}, d_{9}, d_{10}, d_{12}$ & $a_{1}, a_{2}, d_{21}, d_{24}$ & 4 & $\frac{2}{2}=1$ \\
\hline$a_{1}, d_{9}, d_{10}, d_{12}$ & $d_{9}, d_{10}, d_{12}, d_{16}$ & $a_{1}, d_{17}, d_{24}$ & 3 & $\frac{1}{2}$ \\
\hline
\end{tabular}

It is easy to see from formula (37) that when the optimal fractional solution is converted to optimal integer solution, then a type 10 member of the Hilbert basis has a key role. It is $a_{1}, a_{2}, a_{3}, a_{4}, 2 \times d_{24}$.

\section{Conclusions}

This paper continues the analysis of the BCC grids started in [22]. It generalizes the grids to 4 and higher dimensions. Minimal routes are determined from the origin to a target point. An integer linear programming model is applied. Its linear programming relaxation is solved by the simplex method. There is only one case when the optimal solution is 
fractional. The integrality is achieved by the Gomory method in this case. In $4 \mathrm{D}$, Table 1 shows the direct formulae to compute distance based on Theorem 2 (and generalized to $\mathrm{nD}$ in Corollary 1). The non-negative cone of the kernel space of the matrix of the steps in the grid has an important role. The elements of its Hilbert basis describe the alternative routes, i.e., the geometry of the routes, and the changes of the bases during the simplex method.

Author Contributions: Formal analysis, G.K., G.S. and N.D.T.; visualization, B.N.; writing-original draft preparation, B.V.; writing-review and editing, G.K. and B.N. All authors have read and agreed to the published version of the manuscript.

Funding: This research received no external funding.

Conflicts of Interest: The authors declare no conflict of interest.

\section{Appendix A}

Table A1. The bases remained after the filtering of the 4-dimensional case containing no vectors from the first 8 vectors, i.e., containing only diagonal vectors. (The columns of matrix $A$ are referred by their indices from 1 to 24 .)

\begin{tabular}{|c|c|c|c|c|c|c|c|c|c|c|c|}
\hline \multirow{2}{*}{$\frac{\text { No. }}{1}$} & \multicolumn{4}{|c|}{ Basis } & \multirow{2}{*}{$\frac{\text { Optimal }}{\text { YES }}$} & \multirow{2}{*}{$\frac{\text { No. }}{2}$} & \multicolumn{4}{|c|}{ Basis } & \multirow{2}{*}{$\frac{\text { Optimal }}{\text { YES }}$} \\
\hline & 9 & 10 & 11 & 13 & & & 9 & 10 & 11 & 14 & \\
\hline 3 & 9 & 10 & 11 & 15 & YES & 4 & 9 & 10 & 11 & 16 & YES \\
\hline 5 & 9 & 10 & 12 & 13 & YES & 6 & 9 & 10 & 12 & 14 & YES \\
\hline 7 & 9 & 10 & 12 & 15 & YES & 8 & 9 & 10 & 12 & 16 & YES \\
\hline 9 & 9 & 10 & 15 & 19 & NO & 10 & 9 & 10 & 15 & 20 & NO \\
\hline 11 & 9 & 10 & 16 & 19 & NO & 12 & 9 & 10 & 16 & 20 & NO \\
\hline 13 & 9 & 11 & 12 & 14 & YES & 14 & 9 & 11 & 14 & 18 & NO \\
\hline 15 & 9 & 11 & 14 & 20 & $\mathrm{NO}$ & 16 & 9 & 11 & 16 & 18 & NO \\
\hline 17 & 9 & 12 & 13 & 14 & YES & 18 & 9 & 12 & 13 & 18 & NO \\
\hline 19 & 9 & 12 & 13 & 22 & $\mathrm{NO}$ & 20 & 9 & 12 & 14 & 15 & YES \\
\hline 21 & 9 & 12 & 14 & 16 & YES & 22 & 9 & 12 & 14 & 17 & NO \\
\hline 23 & 9 & 12 & 14 & 18 & $\mathrm{NO}$ & 24 & 9 & 12 & 14 & 20 & $\mathrm{NO}$ \\
\hline 25 & 9 & 12 & 14 & 22 & NO & 26 & 9 & 12 & 14 & 23 & $\mathrm{NO}$ \\
\hline 27 & 9 & 12 & 15 & 22 & NO & 28 & 9 & 12 & 16 & 18 & NO \\
\hline 29 & 9 & 12 & 16 & 22 & $\mathrm{NO}$ & 30 & 9 & 14 & 15 & 20 & $\mathrm{NO}$ \\
\hline 31 & 9 & 14 & 16 & 20 & NO & 32 & 9 & 16 & 18 & 19 & NO \\
\hline 33 & 9 & 16 & 18 & 20 & $\mathrm{NO}$ & 34 & 9 & 16 & 20 & 22 & NO \\
\hline 35 & 10 & 11 & 12 & 13 & YES & 36 & 10 & 11 & 13 & 14 & YES \\
\hline 37 & 10 & 11 & 13 & 15 & YES & 38 & 10 & 11 & 13 & 16 & YES \\
\hline 39 & 10 & 11 & 13 & 17 & $\mathrm{NO}$ & 40 & 10 & 11 & 13 & 18 & NO \\
\hline 41 & 10 & 11 & 13 & 19 & $\mathrm{NO}$ & 42 & 10 & 11 & 13 & 21 & $\mathrm{NO}$ \\
\hline 43 & 10 & 11 & 13 & 24 & NO & 44 & 10 & 11 & 14 & 17 & NO \\
\hline 45 & 10 & 11 & 14 & 21 & $\mathrm{NO}$ & 46 & 10 & 11 & 15 & 17 & $\mathrm{NO}$ \\
\hline 47 & 10 & 11 & 15 & 21 & $\mathrm{NO}$ & 48 & 10 & 11 & 16 & 21 & $\mathrm{NO}$ \\
\hline 49 & 10 & 12 & 13 & 17 & $\mathrm{NO}$ & 50 & 10 & 12 & 13 & 19 & $\mathrm{NO}$ \\
\hline 51 & 10 & 12 & 15 & 17 & NO & 52 & 10 & 13 & 15 & 19 & NO \\
\hline 53 & 10 & 13 & 16 & 19 & NO & 54 & 10 & 15 & 17 & 19 & NO \\
\hline 55 & 10 & 15 & 17 & 20 & NO & 56 & 10 & 15 & 19 & 21 & NO \\
\hline 57 & 11 & 12 & 13 & 18 & $\mathrm{NO}$ & 58 & 11 & 12 & 14 & 17 & NO \\
\hline 59 & 11 & 13 & 14 & 18 & NO & 60 & 11 & 13 & 16 & 18 & NO \\
\hline 61 & 11 & 14 & 17 & 18 & NO & 62 & 11 & 14 & 17 & 20 & NO \\
\hline 63 & 11 & 14 & 17 & 21 & NO & 64 & 12 & 13 & 14 & 17 & NO \\
\hline 65 & 12 & 13 & 17 & 18 & $\mathrm{NO}$ & 66 & 12 & 13 & 17 & 22 & NO \\
\hline 67 & 12 & 13 & 18 & 19 & NO & 68 & 12 & 14 & 15 & 17 & $\mathrm{NO}$ \\
\hline
\end{tabular}

\section{References}

1. Klette, R.; Rosenfeld, A. Digital Geometry—Geometric Methods for Digital Picture Analysis; Morgan Kaufmann, Elsevier Science B.V.: Amsterdam, The Netherlands, 2004.

2. Rosenfeld, A.; Pfaltz, J.L. Distance functions on digital pictures. Pattern Recognit. 1968, 1, 33-61. [CrossRef]

3. Borgefors, G. Distance transformations in digital images. Comput. Vision Graph. Image Process. 1986, 34, 344-371. [CrossRef]

4. Csébfalvi, B. Prefiltered Gaussian Reconstruction for High-Quality Rendering of Volumetric Data sampled on a Body-Centered Cubic Grid. In Proceedings of the VIS 05-IEEE Visualization 2005, Minneapolis, MN, USA, 23-28 October 2005; pp. 311-318.

5. Csébfalvi, B. An Evaluation of Prefiltered B-Spline Reconstruction for Quasi-Interpolation on the Body-Centered Cubic Lattice. IEEE Trans. Vis. Comput. Graph. 2010, 16, 499-512. [CrossRef] [PubMed]

6. Vad, V.; Csébfalvi, B.; Rautek, P.; Gröller, M.E. Towards an Unbiased Comparison of CC, BCC, and FCC Lattices in Terms of Prealiasing. Comput. Graph. Forum 2014, 33, 81-90. [CrossRef]

7. Strand, R.; Nagy, B. Path-Based Distance Functions in $n$-Dimensional Generalizations of the Face- and Body-Centered Cubic Grids. Discret. Appl. Math. 2009, 157, 3386-3400. [CrossRef] 
8. Kovács, G.; Nagy, B.; Vizvári, B. Weighted Distances and Digital Disks on the Khalimsky Grid-Disks with Holes and Islands. J. Math. Imaging Vis. 2017, 59, 2-22. [CrossRef]

9. Remy, E.; Thiel, E. Computing 3D Medial Axis for Chamfer Distances. In Proceedings of the DGCI 2000: Discrete Geometry for Computer Imagery-9th International Conference, Lecture Notes in Computer Science, Uppsala, Sweden, 13-15 December 2000; Volume 1953, pp. 418-430.

10. Sintorn, I.-M.; Borgefors, G. Weighted distance transforms in rectangular grids. In Proceedings of the ICIAP, Palermo, Italy, 26-28 September 2001; pp. 322-326.

11. Butt, M.A.; Maragos, P. Optimum design of chamfer distance transforms. IEEE Trans. Image Process. 1998, 7, 1477-1484. [CrossRef] [PubMed]

12. Celebi, M.E.; Celiker, F.; Kingravi, H.A. On Euclidean norm approximations. Pattern Recognit. 2011, 44, 278-283. [CrossRef]

13. Kovács, G.; Nagy, B.; Vizvári, B. On disks of the triangular grid: An application of optimization theory in discrete geometry. Discret. Appl. Math. 2020, 282, 136-151. [CrossRef]

14. Kovács, G.; Nagy, B.; Vizvári, B. Chamfer distances on the isometric grid: A structural description of minimal distances based on linear programming approach. J. Comb. Optim. 2019, 38, 867-886. [CrossRef]

15. Ahuja, R.K.; Magnanti, T.L.; Orlin, J.B. Network Flows: Theory, Algorithms and Applications; Prentice Hall: Upper Saddle River, NJ, USA, 1993.

16. Dantzig, G.B. Programming in a Linear Structure, Report of the September 9, 1948 meeting in Madison. Econometrica 1949, 17, 73-74.

17. Prékopa, A. Lineáris Programozás I; Hungarian, Linear Programming I; Bolyai János Matematikai Társulat (János Bolyai Mathematical Society): Budapest, Hungary, 1968.

18. Hilbert, D. Über die Theorie der algebrischen Formen. Math. Ann. 1890, 36, 473-534. [CrossRef]

19. Jeroslow, R.G. Some basis theorems for integral monoids. Math. Oper. Res. 1978, 3, 145-154.

20. Schrijver, A. On total dual integrality. Linear Algebra Its Appl. 1981, 38, 27-32. [CrossRef]

21. Nagy, B. Union-Freeness, Deterministic Union-Freeness and Union-Complexity. In Proceedings of the DCFS 2019: Descriptional Complexity of Formal Systems-21st IFIP WG 1.02, International Conference, Lecture Notes in Computer Science, Kosice, Slovakia, 17-19 July 2019; Volume 111612, pp. 46-56.

22. Kovács, G.; Nagy, B.; Stomfai, G.; Turgay, N.D.; Vizvári, B. On Chamfer Distances on the Square and Body-Centered Cubic Grids-An Operational Research Approach. 2021. Unpublished work. 